BULLETIN OF THE

AMERICAN MATHEMATICAL SOCIETY

Volume 80, Number 3, May 1974

\title{
COLIMITS IN TOPOI
}

BY ROBERT PARÉ

Communicated by Saunders Mac Lane, September 22, 1973

Given a cartesian closed category $\boldsymbol{E}$ with subobject classifier $t: 1 \rightarrow \Omega$, it is shown that the functor $\Omega^{(1)}: \boldsymbol{E}^{\mathrm{op}} \rightarrow \boldsymbol{E}$ is tripleable. Standard results from the theory of triples are then used to show that $\boldsymbol{E}$ has $I$-colimits if and only if it has $I^{\text {op }}$-limits. This gives a new proof of Mikkelsen's theorem which states that $\boldsymbol{E}$ has all finite colimits.

1. Preliminaries on topoi. A category $\boldsymbol{E}$ is called an elementary topos in [6] if $\boldsymbol{E}$ is cartesian closed and has a subobject classifier $t: 1 \rightarrow \Omega$. The reader who is not familiar with these notions is referred to [3], [4], and [6] (in [3] and [4], the existence of finite limits and colimits is assumed, but we do not make that assumption here). Throughout this paper $\boldsymbol{E}$ will be an elementary topos.

$\boldsymbol{E}$ has finite limits since the existence of binary products and terminal object is assumed in cartesian closedness, and the equalizer of $f, g: A \rightarrow B$ can be constructed as the subobject classified by $A \rightarrow^{(f, g)} B \times B \rightarrow^{\delta} \Omega$ where $\delta$ is the characteristic morphism of the diagonal $\Delta: B \longmapsto B \times B$.

For any object $A$ of $\boldsymbol{E}$ the evaluation morphism $e v_{A}: \Omega^{A} \times A \rightarrow \Omega$ is the characteristic morphism of a subobject $\varepsilon_{A^{\succ}} \rightarrow \Omega^{A} \times A$ called the membership relation.

If $a: A^{\prime} \longmapsto A$ is a monomorphism in $\boldsymbol{E}$, we get another monomorphism

$$
\varepsilon_{A^{\prime}} \longmapsto \Omega^{A^{\prime}} \times A^{\prime}>\Omega^{A^{\prime} \times a} \longrightarrow \Omega^{A^{\prime}} \times A
$$

whose characteristic morphism $\Omega^{A^{\prime}} \times A \rightarrow \Omega$ corresponds, by exponential adjointness, to a morphism $\Omega^{A^{\prime}} \rightarrow \Omega^{A}$ which is denoted $\exists_{a}$ and called the direct image morphism.

The following lemma is fundamental.

LemMa. Let

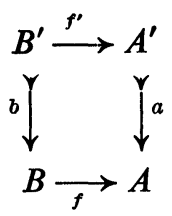

AMS (MOS) subject classifications (1970). Primary 18A30, 18C15, $18 \mathrm{D} 99$.

Key words and phrases. Elementary topos, limits, colimits, triple.

Copyright @ American Mathematical Society 1974 
be a pullback diagram in $\boldsymbol{E}$ and assume that $a$ and $b$ are monomorphisms. Then the following diagram commutes:

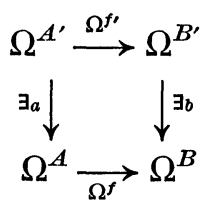

Proof outline. These two morphisms $\Omega^{A^{\prime}} \rightarrow \Omega^{B}$ are equal if and only if their transposes under exponential adjointness, $\Omega^{A^{\prime}} \times B \rightarrow \Omega$ are equal and this is true if and only if the subobjects classified by these two morphisms are equal. This follows from naturality of the evaluation morphisms and the hypothesis of the Lemma.

REMARK. Once the fact that a topos has finite colimits is established, we have image factorizations, and the morphism $\exists_{a}$ can be defined for any morphism $a$ (not only monomorphisms) (see [6]). Then the above Lemma is valid without the restriction that $a$ and $b$ be monomorphisms. This fundamental Lemma, along with the corresponding one with $\exists$ replaced by $\forall$, appears in [11]. The one with $\exists$ appears, in an external form, in $[4$, p. 36] where it is called the Beck condition.

COROLlary. If $a: A^{\prime} \longrightarrow A$ is a monomorphism then

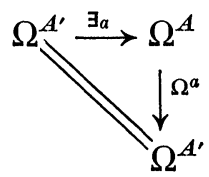

commutes.

Proof. If $a$ is a monomorphism then

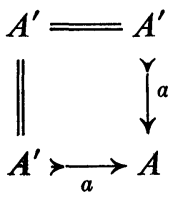

is a pullback diagram. The result follows from the fact that $\exists_{A}{ }^{\prime}=\Omega^{A^{\prime}}$ and the Lemma.

2. Tripleableness. For an introduction to the theory of triples the reader is referred to [2, Introduction], and for further details to [8] (where triples are called monads) and [9].

Recall that a pair of morphisms $b, b^{\prime}: B \rightarrow B^{\prime}$ in a category $B$ is called a reflexive pair (see [7]) if there is a morphism $d: B^{\prime} \rightarrow B$ such that $b d=$ $b^{\prime} d=B^{\prime}$. 
The following slight modification of the Barr-Beck "crude tripleableness theorem" is contained implicitly in [1].

RTT. Let $\boldsymbol{B} \rightarrow{ }^{U} \boldsymbol{A}$ have a left adjoint. If $\boldsymbol{B}$ has coequalizers of reflexive pairs, $U$ preserves these coequalizers, and $U$ reflects isomorphisms, then $U$ is tripleable.

If $E$ is an elementary topos the functor $\Omega^{(~)}: E^{\text {op }} \rightarrow E$ has a left adjoint, namely $\Omega^{()}$itself, as can be seen from the following sequence of natural isomorphisms of hom sets:

$$
\boldsymbol{E}\left(A, \Omega^{B}\right) \cong \boldsymbol{E}(A \times B, \Omega) \cong \boldsymbol{E}(B \times A, \Omega) \cong \boldsymbol{E}\left(B, \Omega^{A}\right) \cong \boldsymbol{E}^{\mathrm{op}}\left(\Omega^{A}, B\right) .
$$

The next theorem, which is the main theorem of the paper, is a generalization of the theorem for the category of sets which says that the opposite of the category of sets is tripleable over sets via the contravariant power set functor. The algebras for the triple are the complete atomic boolean algebras, and the equivalence whose existence is asserted by tripleableness is the familiar Stone duality

Lambek and Rattray [5] have also obtained this theorem as well as some of the consequences drawn from it, but their approach is different. Certain parts of this theorem were already known to Mikkelsen. In particular he has studied to what extend Stone duality holds in a topos.

THEOREM. $\Omega^{(1)}: E^{\text {op }} \rightarrow E$ satisfies the hypotheses of the RTT and therefore is tripleable.

Proof. (i) $\boldsymbol{E}^{\text {op }}$ has all coequalizers since $\boldsymbol{E}$ has all equalizers.

(ii) Let

$$
A \underset{g}{\stackrel{f}{\longrightarrow}} B \stackrel{h}{\longrightarrow} C
$$

be a coequalizer in $\boldsymbol{E}^{\mathrm{op}}$ where $(f, g)$ is a reflexive pair (i.e. there exists $d: B \rightarrow A$ in $E^{\mathrm{op}}$ such that $f d=g d=B$ ). In $E$, this means that

$$
C \stackrel{h}{\longrightarrow} B \underset{g}{\stackrel{f}{\longrightarrow}} A
$$

is an equalizer diagram and $d: A \rightarrow B$ is such that $d f=d g=B$.

It is easily seen that

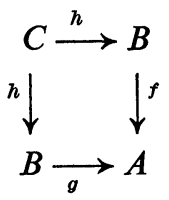

is a pullback diagram. For if $f \phi=g \psi$ then $d f \phi=d g \psi$ so $\phi=\psi$. Then since $h$ is the equalizer of $f$ and $g$, there exists a unique $\theta$ such that $h \theta=\phi=\psi$. 
Also $f, g, h$ are all monomorphisms, $h$ because it is an equalizer and $f$ and $g$ because they are split by $d$. Therefore, by the Lemma and its corollary the following diagrams commute

Therefore
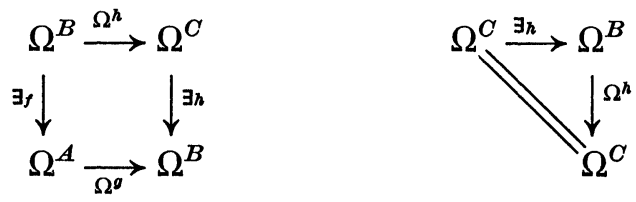

$$
\Omega^{A} \underset{\Omega^{o}}{\stackrel{\Omega^{f}}{\longrightarrow}} \Omega^{B} \stackrel{\Omega^{h}}{\longrightarrow} \Omega^{C}
$$

is not only a coequalizer but a contractible coequalizer (see [1] or [9]).

(iii) Let

$$
B \underset{g}{\stackrel{f}{\longrightarrow}} A
$$

be any two morphisms in $E$ such that $\Omega^{f}=\Omega^{g}$. If $\{\cdot\}: A \rightarrow \Omega^{A}$ is the singleton morphism (i.e. the transpose under exponential adjointness of the characteristic morphism of the diagonal $\Delta: A \longmapsto A \times A)$, then both composites

$$
A \stackrel{\{\cdot\}}{\longrightarrow} \Omega^{A} \underset{\Omega^{o}}{\stackrel{\Omega^{f}}{\longrightarrow}} \Omega^{B}
$$

are equal. Now, taking the subobjects classified by the transposed morphisms, we get $B r^{(f, B)} A \times B$ and $B r^{(g, B)} A \times B$ corresponding to the top and bottom morphisms respectively. Since these subobjects must be equal, we see that $f=g$. Thus $\Omega^{()}$is faithful.

The proof is finished when we note that faithful functors reflect monomorphisms and epimorphisms and an elementary topos is balanced (since any monomorphism is the equalizer of its characteristic morphism and "true") therefore $\Omega^{(\text {" }}$ reflects isomorphisms.

REMARK. If a functor $V: \boldsymbol{E} \rightarrow \boldsymbol{A}$ has a left adjoint and reflects isomorphisms, then $\boldsymbol{E}^{\text {op }} \rightarrow^{\left.\Omega^{\prime}\right)} \boldsymbol{E} \rightarrow^{V} \boldsymbol{A}$ satisfies the conditions of the RTT and is therefore tripleable. Indeed, the only doubtful fact is that $V \Omega^{(1)}$ preserves coequalizers of reflexive pairs, but $\Omega^{()}$takes such a coequalizer in $E^{\text {op }}$ to a contractible coequalizer in $\boldsymbol{E}$, and this, $V$ must preserve.

3. Consequences. The following corollary follows immediately from the well-known fact that tripleable functors create limits (see [9]).

Corollary 1. Let I be a diagram category. If $\boldsymbol{E}$ has $\boldsymbol{I}^{\text {op }}$-limits then $\boldsymbol{E}$ has I-colimits.

In particular we get Mikkelsen's theorem [10]: $\boldsymbol{E}$ has all finite colimits. 
Let $\boldsymbol{E}$ and $\boldsymbol{E}^{\prime}$ be elementary topoi. Let us say that a functor $G: \boldsymbol{E} \rightarrow \boldsymbol{E}^{\prime}$ preserves "power sets" if for every $A$ in $E, G\left(\Omega^{A}\right)$ is naturally isomorphic to $\Omega^{\prime G A}$.

Corollary 2. If $G: \boldsymbol{E} \rightarrow \boldsymbol{E}^{\prime}$ preserves "power sets" then the following hold:

(i) If $G$ has a left adjoint then it also has a right adjoint.

(ii) If $G$ preserves $\boldsymbol{I}^{\mathrm{o}}$-limits then it also preserves $\mathbf{I}$-colimits.

(iii) $G$ preserves equalizers of coreflexive pairs.

Proof. To say that $G$ preserves "power sets" means that

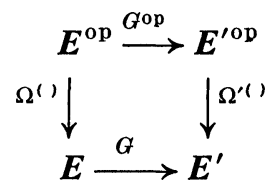

commutes up to isomorphism, i.e. $G^{\mathrm{op}}$ is a $G$-algebraic functor in the terminology of [9]. By a theorem on the existence of left adjoints for such algebraic functors [9] we conclude that (i) holds.

Since $\Omega^{()}$and $\Omega^{\prime \prime)}$ create all limits, then $G^{\text {op }}$ preserves whatever type of limits $G$ preserves. This gives us (ii).

Finally $G^{\text {op }}$ preserves coequalizers of reflexive pairs since $\Omega^{(\text {) }}$ sends them to contractible coequalizers which $G$ necessarily preserves, and $\Omega^{\prime()}$ reflects such coequalizers.

Assume that $U: B \rightarrow \boldsymbol{A}$ is tripleable and that $\boldsymbol{B}$ has coequalizers of reflexive pairs. Then a slight refinement of a theorem of Linton's [7, p. 81] says that if $\boldsymbol{A}$ has $\boldsymbol{I}$-colimits then so does $\boldsymbol{B}$. If $F$ is the left adjoint of $U$ with counit $\varepsilon: F U \rightarrow B$, then colim $\Phi$ can be computed as the coequalizer of the reflexive pair

$$
F \operatorname{colim} U F U \Phi \underset{\varepsilon F \operatorname{colim} U \Phi}{\stackrel{F \operatorname{colim} U \varepsilon \Phi}{\longrightarrow}} F \operatorname{colim} U \Phi \text {. }
$$

Corollary 3. If $\boldsymbol{E}$ has $\mathbf{I}$-colimits then $\boldsymbol{E}$ has $\boldsymbol{I}^{\text {op }}$-limits.

The above construction applied to the case under consideration shows us that in $\boldsymbol{E}, \boldsymbol{I}^{\mathrm{op}}$-limits can be calculated by taking $\boldsymbol{I}$-colimits, applying $\Omega^{()}$, and then taking an equalizer of a coreflexive pair. To say that a functor $G: \boldsymbol{E} \rightarrow \boldsymbol{E}^{\prime}$ preserves "power sets" means that it commutes with $\Omega^{()}$, and Corollary 2(iii) says that $G$ preserves equalizers of coreflexive pairs. Thus we get the following corollary.

COROLlaRY 4. Let $\boldsymbol{G}: \boldsymbol{E} \rightarrow \boldsymbol{E}^{\prime}$ be a functor which preserves "power sets". If $G$ preserves $\mathbf{I}$-colimits then $G$ preserves $I^{\text {op }}$-limits. 


\section{REFERENCES}

1. J. Beck, The tripleableness theorem, unpublished manuscript, Cornell University, Ithaca, N.Y., 1967.

2. B. Eckmann, Seminar on triples and categorical homology theory (ETH 1966/67), Lecture Notes in Math., no. 80, Springer-Verlag, Berlin and New York, 1969. MR 39 \#1511.

3. P. Freyd, Aspects of topoi, Bull. Austral. Math. Soc. 7 (1972), 1-76.

4. A. Kock and G. Wraith, Elementary toposes, Aarhus Lecture Notes, no. 30, 1971.

5. J. Lambek and B. A. Rattray, Localization and sheaf reflectors (to appear).

6. F. W. Lawvere, Introduction in: Toposes, algebraic geometry and logic, Lecture Notes in Math., no. 274, Springer, Berlin, 1972, pp. 1-12.

7. F. E. J. Linton, Coequalizers in categories of algebras, Seminar on Triples and Categorical Homology Theory, Lecture Notes in Math., no. 80, Springer, Berlin, 1969, pp. 75-90.

8. S. Mac Lane, Categories for the working mathematician, Graduate Texts in Math., no. 5, Springer, Berlin, 1971.

9. E. G. Manes, A triple miscellany: Some aspects of the theory of algebras over a triple, Dissertation, Wesleyan University, Middletown, Conn., 1967.

10. C. J. Mikkelsen, Finite colimits in toposes, Talk at the conference on category theory at Oberwolfach, July 1972.

11. M. Tierney, Seminar on topoi, Dalhousie University, 1969-70.

Department of Mathematics, Dalhousie University, Halifax, Nova Scotia, Canada 